\title{
High-dose-rate brachytherapy as monotherapy for localized prostate cancer using three different doses - 14 years of single-centre experience
}

\author{
Carlo Pietro Soatti, MD!, Durim Delishaj, MD!, Romerai D’Amico, MD!, Cristina Frigerio, MD², Ilaria Costanza Fumagalli, MD³, \\ Francesco Bonsignore, MD², Giulia Sangalli, MD², Fausto Declich, MD², Stefano Arcangeli, PhD³, Antonio Ardizzoia, MD4, \\ Alessandro Colombo, MD' \\ 'Department of Radiation Oncology, Alessandro Manzoni Hospital, Lecco, Italy, ${ }^{2}$ Medical Physics Unit, Alessandro Manzoni Hospital, Lecco, \\ Italy, ${ }^{3}$ Department of Radiation Oncology, Policlinico S. Gerardo and University of Milan "Bicocca", Milan, Italy, "Department of Oncology, \\ Alessandro Manzoni Hospital, Lecco, Italy
}

\begin{abstract}
Purpose: To evaluate clinical outcomes in patients with localized prostate cancer (LPC) treated with 3D conformal high-dose-rate (HDR) brachytherapy (BT) as monotherapy.

Material and methods: From March 2004 to November 2017, 277 men with LPC underwent 3D conformal HDR-BT as monotherapy, with a temporary implant. The dose prescription was: 38 Gy in 4 fractions (149 patients), 27 Gy in 2 fractions (41 patients), and 19-20 Gy in a single fraction (87 patients). Biochemical progression-free survival (bPFS), progression-free survival (PFS), and cancer-specific survival (CSS) were calculated. Acute and late genitourinary (GU) and gastrointestinal (GI) toxicity assessment were performed using Common Terminology Criteria for Adverse Events v5.0.

Results: The mean age was 67 (range, 47-81) years. Overall, 145 patients were low-risk, 116 intermediate-risk, and 16 high-risk prostate cancer. After a median follow-up of six years (range, 6-160 months), bPFS, PFS, and CSS were $81 \%, 96 \%$, and $97 \%$, respectively. Dose prescription, initial prostate specific antigen (iPSA) $\geq 9,5 \mathrm{ng} / \mathrm{ml}$, and high-risk disease resulted in prognostic factors regarding bPFS. Only G2-G3 acute or late GI and GU toxicities were observed.

Conclusions: HDR-BT as monotherapy is a valid and safe treatment modality for localized prostate cancer. After a long follow-up, patients receiving 19-20 Gy in a single fraction had a lower biochemical control rate compared to patients receiving $38 \mathrm{~Gy}$ in 4 fractions or $27 \mathrm{~Gy}$ in 2 fractions. Randomized prospective trials with a longer follow-up are necessary to confirm our results, and define total doses and dose per fraction for HDR-BT in patients with LPC.

J Contemp Brachytherapy 2020; 12, 6: 533-539 DOI: https://doi.org/10.5114/jcb.2020.101685
\end{abstract}

Key words: high-dose-rate, brachytherapy, prostate cancer, prostate-specific antigen, PSA.

\section{Purpose}

Prostate cancer is the most common solid tumor in men [1]. With the introduction of serum prostate-specific antigen (PSA) testing, the incidence of prostate cancer has increased in recent years [2]. Changes in screening recommendations indicate that about $90 \%$ of patients are diagnosed with localized prostate cancer (LPC) [3].

There are different modalities for radical treatment of LPC, including radical prostatectomy, external beam radiotherapy (EBRT), and brachytherapy (BT) [4].

Brachytherapy treatment can be delivered by highdose-rate (HDR) or low-dose-rate (LDR), and can deliver a higher radiation dose to the target, avoiding surrounding tissues. The use of high doses per fraction has a bio- logical dose advantage for tumors with a low $\alpha / \beta$ ratio, such as prostate cancer. A higher dose per fraction regimen leads to an increased tumor control. Due to these characteristics, BT is commonly the primary treatment for LPC and achieves excellent outcomes in terms of disease control and toxicities $[5,6,7]$.

High-dose-rate BT has various technical advantages over LDR. For example, needle catheters can be placed outside of the prostate gland, which allows for improved coverage in cases of extracapsular extension or seminal vesicle invasion. Moreover, by using HDR-BT techniques, radioactive source dwell-time positions can be programmed directly by the physician. This increases the dose to tumor area and reduces the dose to organs at risk, including the urethra and rectum $[8,9]$.

\footnotetext{
Address for correspondence: Durim Delishaj, MD, Department of Radiotherapy, Alessandro Manzoni Hospital, Via dell'Esremo, 9/11, Lecco, Italy, phone: +39 0341253540, fax: +39 0341489720, 
Different studies have reported the results of HDR-BT as a monotherapy for the treatment of LPC, confirming its efficacy and low toxicity $[10,11,12,13,14,15,16,17,18,19,20]$.

Table 1. Patient and tumor characteristics

\begin{tabular}{|c|c|c|}
\hline Characteristic & $n$ & $\%$ \\
\hline All patients & 277 & \\
\hline \multicolumn{3}{|l|}{ Age (years) } \\
\hline Median & 67 & \\
\hline Range & $47-81$ & \\
\hline \multicolumn{3}{|l|}{ Gleason score } \\
\hline$\leq 6$ & 179 & 64.6 \\
\hline 7 & 91 & 32.9 \\
\hline 8 & 7 & 2.5 \\
\hline \multicolumn{3}{|l|}{ iPSA (ng/ml) } \\
\hline Median & 7.85 & \\
\hline Range & $1.8-59.5$ & \\
\hline \multicolumn{3}{|c|}{ T stage (DRE or image- based) } \\
\hline T1c & 217 & 78.3 \\
\hline $\mathrm{T} 2 \mathrm{a}$ & 54 & 19.5 \\
\hline $\mathrm{T} 2 \mathrm{~b}$ & 3 & 1.1 \\
\hline $\mathrm{T} 2 \mathrm{C}$ & 3 & 1.1 \\
\hline \multicolumn{3}{|l|}{ NADT } \\
\hline Yes & 94 & 33.9 \\
\hline No & 183 & 66.1 \\
\hline \multicolumn{3}{|l|}{ Risk group } \\
\hline Low & 145 & 52 \\
\hline Intermediate & 116 & 42 \\
\hline High & 16 & 6 \\
\hline \multicolumn{3}{|c|}{ Positive biopsy cores (\%) } \\
\hline Median & & 30 \\
\hline Range & & $5-100$ \\
\hline \multicolumn{3}{|l|}{ HDR-BT dose } \\
\hline 19-20 Gy/1 fraction & 87 & 31.4 \\
\hline 27 Gy/2 fractions & 41 & 14.8 \\
\hline 38 Gy/4 fractions & 149 & 53.8 \\
\hline \multicolumn{3}{|l|}{ Adjuvant ADT } \\
\hline Yes & 6 & 2.2 \\
\hline No & 271 & 97.8 \\
\hline
\end{tabular}

NADT - neoadjuvant androgen deprivation therapy, ADT - androgen depriva tion therapy, DRE - digital rectal examination, IPSA - initial prostate-specific antigen, HDR-BT - high-dose-rate brachytherapy
While the use of brachytherapy in prostate cancer is well-known, currently, the total dose and fractionation for HDR-BT in the treatment of localized prostate cancer are not yet established, and finding the optimal HDR brachytherapy schedule remains a challenge.

Our study aimed to retrospectively evaluate clinical outcomes in patients affected by LPC and treated with 3D conformal HDR-BT as monotherapy.

\section{Material and methods}

\section{Patients and tumor characteristics}

From March 2004 to November 2018, a total of 277 men with localized prostate cancer (cT1c-T2cN0M0) were treated in our institute using HDR-BT and ${ }^{192}$ Ir source.

The data were collected retrospectively by a radiation oncologist. Pre-treatment evaluation included clinical examination, digital rectal examination (DRE), routine pre-operative determination of blood chemistries and blood counts, PSA, Gleason grade, and computed tomography $(\mathrm{CT})$ or magnetic resonance imaging (MRI) in selected cases.

After HDR-BT treatment, all patients continued regular follow-up in our institution, with clinical examination, DRE, biochemical control, and additional radiologic tests if clinically indicated. Gastrointestinal and urinary toxicities was evaluated at the clinical examination according to the National Cancer Institute Common Toxicity Criteria for Adverse Events version 5 of 2017.

All patients, after histologically confirmed prostate cancer, were stratified into low-risk prostate cancer (stage T1-T2a, Gleason score [GS] $\leq 6$, and PSA $\leq 10$ ), intermediate-risk prostate cancer (stage T2b-T2c and/or GS $=7$, and/or PSA > 10-20), and high-risk prostate cancer (stage $>\mathrm{T} 2 \mathrm{c}$ and/or GS $=8-10$, and/or PSA $>20$ ) according to D'Amico et al. and the EAU criteria [21,22]. The median age was 67 (range, 47-81) years.

Of them, the majority of patients $(94.6 \%)$ were lowand intermediate-risk (145 patients were low-risk and 116 intermediate-risk), and only 5.7\% resulted high-risk (16 patients). Overall, neoadjuvant androgen deprivation was administered in $33.9 \%$ of patients for a median of 3 months. Finally, only $2.2 \%$ of patients received adjuvant androgen deprivation therapy (ADT). Patients and tumor characteristics are shown in Table 1.

\section{HDR-BT technique}

After given informed consent, the patients were placed in a lithotomy position and underwent epidural anesthesia. A urinary catheter was placed into urinary bladder. A 7.5-MHz biplanar transrectal ultrasound (TRUS) transducer was inserted into the rectum to identify the prostate and urethra. The applicators were inserted transperineally under direct ultrasound monitoring control. After insertion and immobilization of anchor needles, the flexible applicator needles were implanted from the anterior to the posterior rows in the periphery of prostate to minimize rotation. Subsequently, the remaining needles for the internal regions were implanted. The treatment plan was done using Oncentra Prostate software to perform 3D 
conformal dose planning supported by an inverse planning algorithm. Clinical target volume (CTV) was defined by the whole prostate with a $3 \mathrm{~mm}$ margin. If seminal vesicle invasion was observed or diagnosed by imaging, the applicator needles were placed with the seminal in the CTV. The planning target volume was defined as equal to the CTV. Dosimetric goals included plaining aim dose to the target was dose $\left(D_{90}\right) 90 \%>95 \%$, dose received by 2cc rectum $\left(\mathrm{D}_{2 \mathrm{cc}}\right)<75 \%$ of prescription dose $(\mathrm{PD})$, and $\mathrm{D}_{2 \mathrm{cc}}$ bladder $<80 \%$ of prescribed dose. Finally, urethra dosimetric goals included the dose received by $1 \%$ volume $\left(\mathrm{V}_{1}\right)<115 \%$ PD and $10 \%$ volume $\left(\mathrm{V}_{10}\right)<110 \%$ PD.

According to NCCN [4] and ABS guidelines [7], following the evolution of knowledge in HDR-BT as well as usage of a new regimen of fractionation published in the literature and our institutional experience, the total doses were prescribed as follow: $38 \mathrm{~Gy}$ in four fractions in 149 patients (period 2004-2010), 27 Gy in two fractions in 41 patients (period 2010-2013), and 19-20 Gy in single fraction in 87 patients (period 2014-2017). In patients receiving $38 \mathrm{~Gy}$, the four fractions were delivered twice daily, with a minimum interval of 6 hours, while in patients receiving $27 \mathrm{~Gy}$, the two fractions were delivered with an interval of 1 or 2 weeks. The treatment was performed using the MicroSelectron ${ }^{\circledR}$ digital HDR (Elekta AB v2).

\section{Statistical analyses}

Before performing statistical analysis, an exploration phase was carried out; categorical data were described by frequency, whereas continuous data by a mean and median.

Biochemical progression was defined as post-treatment PSA greater than 2 units above the nadir value.

A

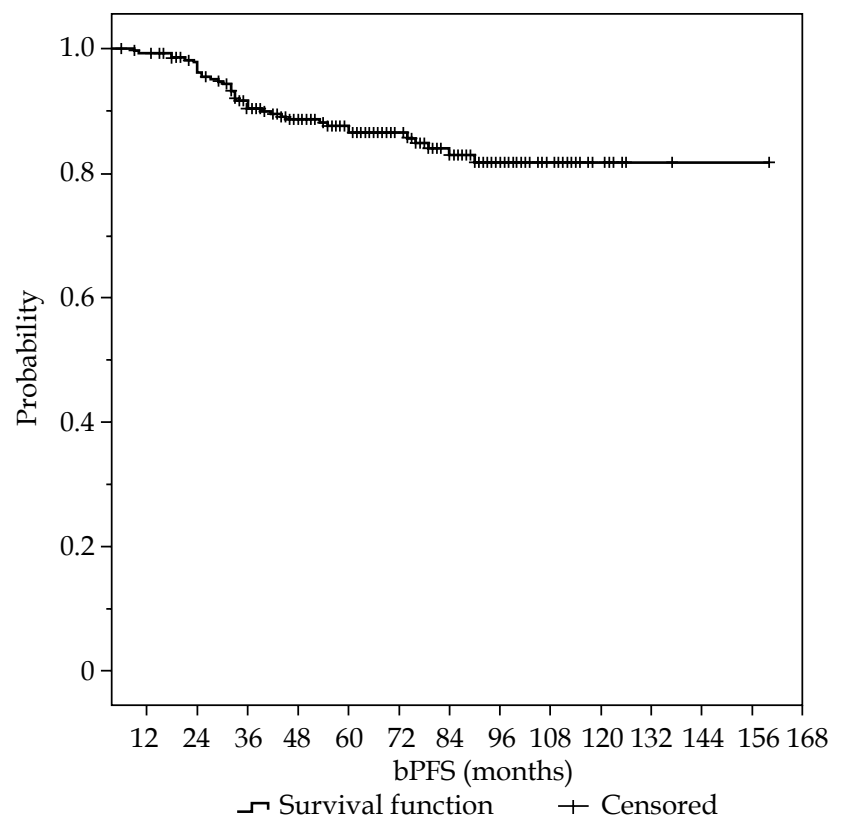

Fig. 1. Kaplan-Meier curves of biochemical progression-free survival A) in all patients and B) in patients treated with 19-20 Gy in single fraction, 27 in two fractions, and $38 \mathrm{~Gy}$ in four fractions
Progression-free survival (PFS) was defined as the time from the HDR-BT treatment to the date of occurrence of any of the following events: local recurrence, local treatment (surgery, re-irradiation), or identification of distant metastasis.

Survival functions and disease control were calculated using the Kaplan-Meier method, and the log-rank test was used to evaluate the differences between curves.

Overall survival was calculated from the date of HDR-BT treatment to the date of death. Cancer-specific survival was defined as the interval of time from BT treatment to the date of cancer-related death.

Univariate analysis was performed including each risk factor in a Cox regression model. The results of the Cox regression were expressed by hazard ratios (HR), with its related confidence interval (CI) and related $p$-value calculated using a Wald test. Differences were considered significant at $p<0.05$. Analyses were performed with a SPSS 22 software data analyses.

\section{Results}

After a median follow-up period of 6 years (range, 6-160 months), the biochemical progression-free survival (bPFS) and PFS were $81 \%$ and $96 \%$, respectively. After 3,5 , and 8 years, the bPFS rates were $92 \%, 85 \%$, and $81 \%$, respectively. Figure 1A and B shows the Kaplan-Meier curves regarding bPFS.

Data analyses showed that the median initial prostate specific antigen (iPSA) was $7.85 \mathrm{ng} / \mathrm{ml}$, ranging from 1.8 to $59.5 \mathrm{ng} / \mathrm{ml}$.

Subgroup analysis based on dose prescription showed that in patients treated with a total dose of $38 \mathrm{~Gy}$ in four fractions, the bPFS was $91 \%$. In patients receiving $27 \mathrm{~Gy}$ in

B

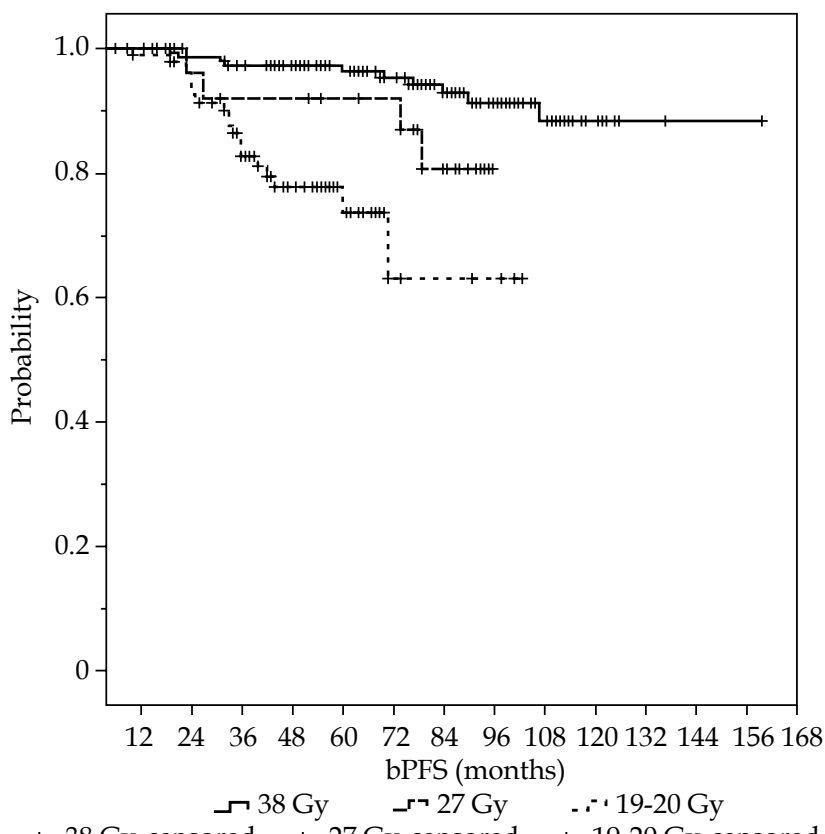

+ 38 Gy-censored + 27 Gy-censored + 19-20 Gy-censored 
A

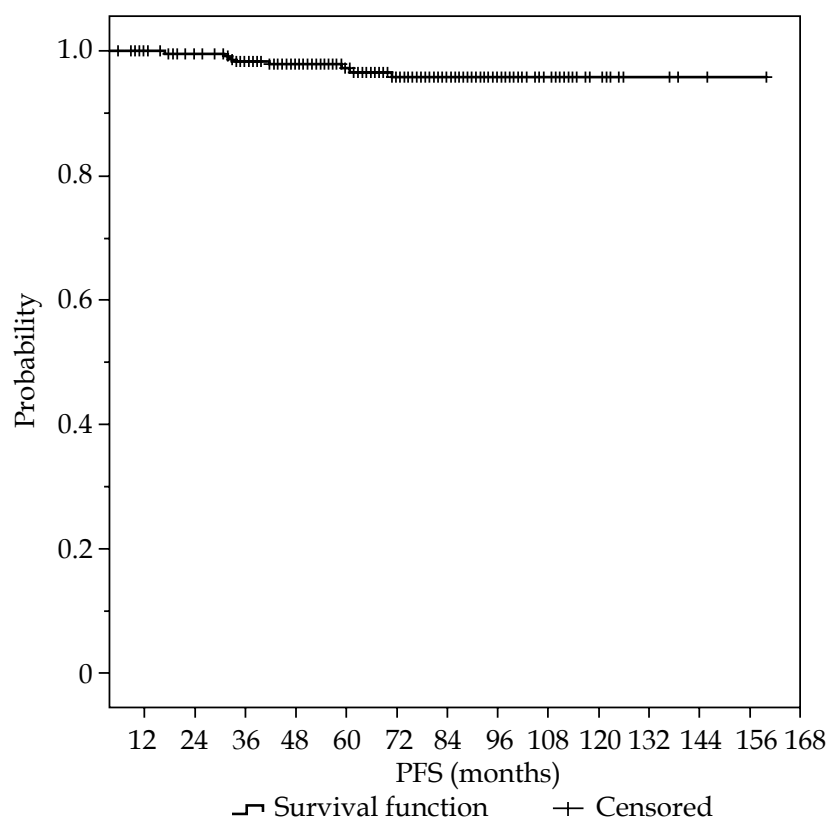

B

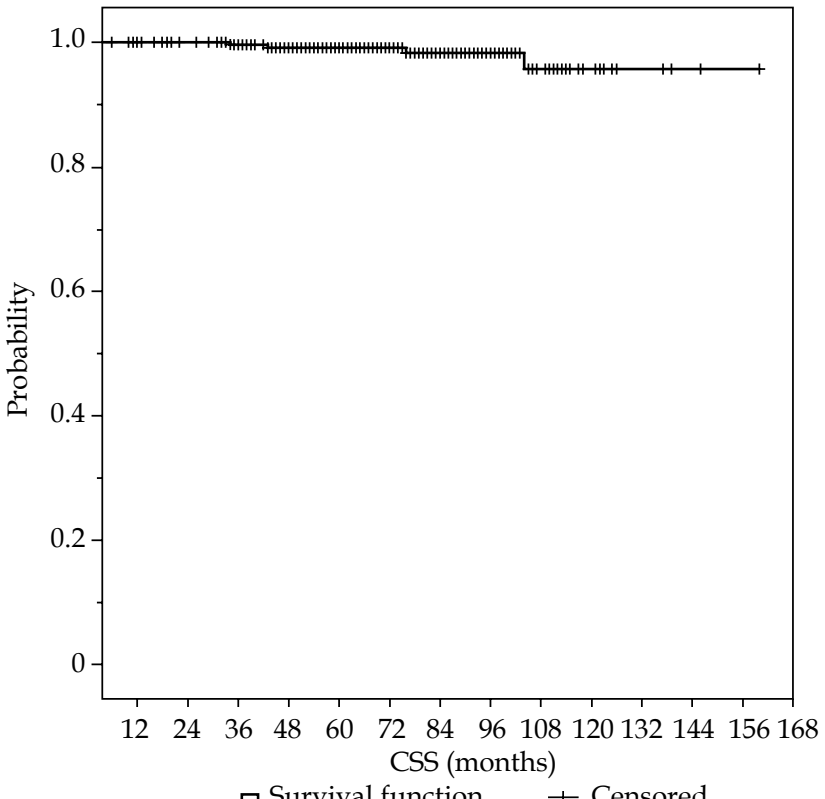

Fig. 2. Kaplan-Meier curves of progression-free survival (A) and cancer-specific survival of all patients analyzed (B)

two fractions, the bPFS was $86 \%$, whilst in patients treated with 19-20 Gy in a single fraction, the bPFS was $65 \%$.

Univariate analysis showed that patients receiving 38 Gy in four fractions or 27 Gy in two fractions had a statically significant advantage in terms of bPFS (Figure 2B) compared to those treated with a dose of 19-20 Gy in single fraction $(p$-value $=0.001, \mathrm{HR}=1.821,95 \% \mathrm{CI}=$ 1.239-2.379). There was no statistically significant difference between patients receiving $38 \mathrm{~Gy}$ in four fractions compared to patients treated with $27 \mathrm{~Gy}$ in two fractions $(p$-value $>0.05)$

As expected, the bPFS was higher in patients classified as low- to intermediate-risk prostate cancer compared to patients classified as high-risk prostate cancer (bPFS rate of $90 \%$ and $76 \%$, respectively). This difference was statistically significant in favor of patients with lowto intermediate-risk prostate cancer ( $p$-value $<0.05$ ).

Subgroup analyses revealed that this advantage was maintained for patients receiving $38 \mathrm{~Gy}$ in four fractions and $27 \mathrm{~Gy}$ in two fractions, but was lost in patients treated with a total dose of 19-20 Gy in a single fraction. This finding can be explained by a higher number of biochemical recurrences in this subset of patients regardless of their prostate cancer risk. Moreover, patients with iPSA $<9.4 \mathrm{ng} / \mathrm{ml}$ had an advantage in terms of bPFS compared to patients with iPSA $\geq 9.5 \mathrm{ng} / \mathrm{ml}(p=0.022$, $\mathrm{HR}=2.042,95 \% \mathrm{CI}=1.123-4.081$ ).

In a subgroup analysis, this advantage was lost in patients receiving 38 Gy in four fractions or 27 Gy in two fractions, and was confirmed in patients treated with a total dose of 19-20 Gy in a single fraction ( $p$-value $=0.0001$ ).

There was no statistically significant difference between patients receiving ADT therapy or not, even in the subgroup analysis of prescribed dose ( $p$-value $>0.05$ ).

Multivariate analysis (Table 2) showed that the total prescription dose and iPSA $>9 \mathrm{ng} / \mathrm{ml}$ maintained statistically significant differences and prognostic factors were confirmed for bPFS ( $p$-value $<0.05)$.

Of 42 patients with recurrences, the majority of patients had loco-regional recurrences $(85 \%)$ and only $15 \%$

Table 2. Univariate and multivariate analysis of prognostic factors of biochemical progression-free survival in patients affected by localized prostate cancer treated with HDR-BT as monotherapy

\begin{tabular}{|c|c|c|c|c|c|c|}
\hline \multirow[t]{2}{*}{ Factor } & \multicolumn{3}{|c|}{ Univariate analysis } & \multicolumn{3}{|c|}{ Multivariate analysis } \\
\hline & $\mathrm{HR}$ & $95 \% \mathrm{Cl}$ & $P$-value & $\mathrm{HR}$ & $95 \% \mathrm{Cl}$ & $P$-value \\
\hline $\begin{array}{l}\text { iPSA } \\
\geq 9.5 \text { vs. }<9.4\end{array}$ & 2.042 & $1.123-4.081$ & 0.022 & 2.581 & $1.194-5.581$ & 0.016 \\
\hline $\begin{array}{l}\text { Disease risk } \\
\text { L vs. I and H }\end{array}$ & 2.453 & $1.3113-4.543$ & 0.04 & 1.353 & $1.621-2.949$ & 0.447 \\
\hline $\begin{array}{l}\text { Dose prescription (Gy) } \\
38 / 4 \text { f or } 27 / 2 \text { f vs. } 19-20 / f\end{array}$ & 6.813 & $3.833-11.981$ & 0.0001 & 1.46 & $1.064-1.332$ & 0.001 \\
\hline $\begin{array}{l}\text { ADT } \\
\text { Yes vs. No }\end{array}$ & 1.27 & $1.642-2.159$ & 0.49 & - & - & - \\
\hline
\end{tabular}

ADT - androgen deprivation therapy, iPSA - initial prostate-specific antigen 
had distant metastases. Of them, 26 patients $(62 \%)$ received salvage ADT, eight patients (19\%) received EBRT treatment, four patients $(9.5 \%)$ underwent salvage surgery, and four patients $(9.5 \%)$ did not receive any salvage therapy, only PSA monitoring.

Overall survival (OS) and cancer-specific survival (CSS) rates were $83 \%$ and $97 \%$, respectively. For survival rate, there was no statistically significant difference between patients treated with 19-20 Gy in a single fraction, $27 \mathrm{~Gy}$ in two fractions, and $38 \mathrm{~Gy}$ in four fractions.

Finally, overall genitourinary (GU) and gastrointestinal (GI) acute toxicities G2-G3 were 28\%. Late G2-G3 GU and GI toxicities were very low $(2.2 \%)$ and of these patients, only three reported G3 late toxicity $(0.8 \%)$, which involved GU toxicity.

\section{Discussion}

The challenge in radiation oncology is to deliver high doses of radiation to the tumor while limiting the dose to surrounding tissue, thereby reducing the risk of toxicity.

It is well-known that a higher dose to the tumor or high-dose per fraction improve the outcomes of radiation therapy in prostate cancer, with acceptable toxicity. Indeed, randomized trials and meta-analyses have shown that a total dose of 78-80 Gy given in a standard regimen and EBRT technique improve a biochemical control for all risk groups $[23,24,25,26,27,28,29,30,31]$.

HDR-BT is commonly used in combination with EBRT or as monotherapy for the treatment of prostate cancer, with excellent outcomes in terms of disease control and toxicities $[30,31,32,33,34,35,36,37,38,39,40]$.

In a comprehensive comparative meta-analysis, Grimm et al. demonstrated that across all risk groups, BT was associated with better bPFS than surgery or EBRT alone [31]. Currently, the total dose and fractionation for HDR-BT in the treatment of LPC are not yet established, and finding the optimal HDR brachytherapy schedule remains a challenge.

For this reason, the American Brachytherapy Society does not recommend a specific dose for fraction schedules [32].

Earlier studies used many small fractions (often six or more) to prevent possible acute or late toxicity of large doses per fraction [15]. Long-term biochemical control rates of over $90 \%$ (with low-rate toxicity $<5 \%$ ) were reported in patients with an intermediate-risk disease using four-six fraction regimens (6.5-7.5 Gy per fraction) [33].

Zamboglou et al. [35] published their results of 718 patients with clinically localized prostate cancer, treated with HDR-BT as monotherapy with a total dose of $38 \mathrm{~Gy}$ in four fractions. After a median follow-up of 4 years, 3- and 8-year biochemical control was $97 \%$ and $94 \%$, respectively. Metastasis-free survival rates were $98 \%$ and $97 \%$, respectively. Similar results were described by Jawad et al. [37] for favorable-risk prostate patients, who underwent HDR brachytherapy as monotherapy at the dose of 38 Gy in four fractions, 24 Gy in two fractions, and 27 Gy in two fractions. Minimal grade 3 toxicities were observed, but no grade 4 or higher.
Recently, some authors reported results of a single fraction of 19 Gy for the treatment of localized prostate cancer with discordant data regarding biochemical control $[38,39,40]$.

In a prospective study from 2017, Krauss et al. published the results of 63 patients with intermediate-risk prostate cancer, who were treated with a dose of 19 Gy single fraction using HDR brachytherapy. After a follow-up of 2.9 years, the biochemical control rate was $93 \%$. No grade 3 urinary toxicities were reported [38]. The limitation of this study was a short follow-up ( $<3$ years).

A higher rate of biochemical failure in patients receiving 19 Gy in single fractions has been described in the literature. In a randomized trial, Morton et al. compared single fraction of $19 \mathrm{~Gy}$ to two fractions of $13.5 \mathrm{~Gy}$ in patients with low- and intermediate-risk disease. Both treatment regimens were very well tolerated, with an acute retention rate of $2.4 \%$ and grade 3 toxicity rate of $<1 \%$. Local recurrence was observed in the single fraction arm only [39].

In our study, after a long follow-up, we found excellent results in terms of bPFS and PFS (82\% and 96\%, respectively), with a superb CSS rate of $97 \%$, despite including patients with high-risk prostate cancer who were unfit for other treatments or refused to have them.

Patients receiving a total dose of 38 Gy in four fractions or 27 Gy in two fractions had a higher biochemical-free progression (90\%) compared with those treated with 19-20 Gy in a single fraction (bPFS 65\%).

There was no statistically significant difference between patients treated with a 19-20 Gy single fraction, $27 \mathrm{~Gy}$ in two fractions, and $38 \mathrm{~Gy}$ in four fractions, regarding PFS and OS.

Our results demonstrate low unacceptable biochemical control rates for patients with localized prostate cancer treated with a single fraction of 19-20 Gy compared to patients receiving $38 \mathrm{~Gy}$ in four fractions or $27 \mathrm{~Gy}$ in two fractions. We did not identify any dosimetric factor associated with the risk of recurrence, which can be described in terms of cancer radiobiology, such as biological effective dose (BED), re-oxygenation, and phases of the cell cycle.

For HDR prostate brachytherapy, simplified-form BED is not appropriate, and full-form BED (considering intrafraction and interfraction repair, repopulation, and time of irradiation) can be considered [40,41,42].

It is estimated that full-form BED for a total dose of $20 \mathrm{~Gy}$ in a single fraction corresponds to only $64-82 \%$ of simplified-form BED calculation, and that regimen with more than a fraction simplified-form BED correspond to 90-94\% of full-form BED [42].

For this reason; the full-form BED calculation seems to be lower in patients treated with a total dose of 19-20 Gy in a single fraction compared to patients receiving $27 \mathrm{~Gy}$ in two fractions and $38 \mathrm{~Gy}$ in four fractions. Moreover, hypoxia is commonly present in prostate cancer, and administering more than one fraction supports the improvement of tumor response, while using a single fraction, the re-oxygenation effect is lost.

Finally, by using a single fraction, the re-distribution effect can be lost because a proportion of cells may be in 
relatively radioresistant phases of the cell cycle, whereas treating with more than one fraction enables the re-distribution into other phases, when the cells are more sensitive [43].

Similar to our findings results were described by Prada et al. [44], where 60 patients with favorable clinical LPC underwent HDR-BT at the dose of 19 Gy in a single fraction. After a median follow-up of 6 years, biochemical control was $66 \%$. The overall and tumor-free survivals were $90 \%$ and $88 \%$, respectively.

Moreover, Morton et al. [45] recently published their data of a randomized phase II clinical trial, where 170 patients with LPC were randomized to receive HDR as either a single fraction of $19 \mathrm{~Gy}$ or as two fractions of $13.5 \mathrm{~Gy}$, one week apart. After a median follow-up of 60 months, 5-year bPFS and cumulative incidence of local failure was $73.5 \%$ and $29 \%$, respectively, in the single fraction arm, and $95 \%(p=0.001)$ and $3 \%(p<0.001)$, respectively, in the two-fraction group. Recurrence was not associated with initial stage, grade group, or risk group. The authors concluded that HDR monotherapy delivered as two fractions of 13.5 Gy was well tolerated, with a high cancer control rate at 5 years. Single fractions of $19-20$ Gy as monotherapy were inferior and were not recommended.

Based on our long follow-up results, a single fraction of 19-20 Gy as a monotherapy was not adequate for biochemical local control of LPC.

Limitations of our study include its retrospective nature, lack of data regarding acute and late toxicity, and difficulty in obtaining a complete data, since the study was not randomized, and many patients have been lost to follow-up or died due to other causes.

Overall, an optimal results in terms of bPFS and PFS ( $81 \%$ and $96 \%$, respectively) were found, with an excellent CSS rate (97\%) after a long follow-up, despite including patients with high-risk prostate cancer unfit for other treatments or who refused other therapies.

\section{Conclusions}

In our experience, HDR-BT as monotherapy is a valid modality for the treatment of LPC in terms of biochemical control, local control, and overall survival.

A total dose of 38 Gy in four fractions or 27 Gy in two fractions, with or without an addition of ADT, was adequate for LPC treatment achieving an excellent biochemical control rate. After a long follow-up, we found lower bPFS in patients receiving 19-20 Gy in single fraction (65\%), whereas 19-20 Gy was not adequate for the treatment of localized prostate cancer as a monotherapy and should only be prescribed in selected cases.

HDR-BT was safe and effective, with very low rates of GU and GI acute and late toxicities.

Finally, randomized trials with a longer follow-up are necessary to confirm our results and to define the total dose and dose per fraction for the treatment of LPC with HDR-BT, considering prostate cancer risk classification.

\section{Disclosure}

The authors report no conflict of interest.

\section{References}

1. Siegel RL, Miller KD, Jemal A. CA Cancer statistics, 2019. Cancer J Clin 2019; 69: 7-34.

2. Pinsky PF, Prorok PC, Yu K et al. Extended mortality results for prostate cancer screening in the PLCO trial with median follow-up of 15 years. Cancer 2017; 123: 592-599.

3. Herget KA, Patel DP, Hanson HA et al. Recent decline in prostate cancer incidence in the United States, by age, stage, and Gleason score. Cancer Med 2016; 5: 136-141.

4. NCCN Clinical Practice Guidelines in Oncology (NCCN Guidelines®). Prostate cancer version 2. 2019. Available at: https://www.nccn.org/professionals/physician_gls/pdf/ prostate.pdf (accessed April 20, 2019).

5. Martinez A, Gonzalez J, Stromberg J et al. Conformal prostate brachytherapy: initial experience of a phase I/II dose-escalating trial. Int J Radiat Oncol Biol Phys 1995; 33: 1019-1027.

6. Yamada Y, Rogers L, Demanes DJ et al. American Brachytherapy Society consensus guidelines for high-dose-rate prostate brachytherapy. American Brachytherapy Society. Brachytherapy 2012; 11: 20-32.

7. Crook J. The role of brachytherapy in the definitive management of prostate cancer. Cancer Radiother 2011; 15: 230-237.

8. Davis BJ, Horwitz EM, Lee WR et al. American Brachytherapy Society consensus guidelines for transrectal ultrasound-guided permanent prostate brachytherapy. Brachytherapy 2012; 11: 6-19.

9. White EC, Kamrava MR, Demarco J et al. High-dose-rate prostate brachytherapy consistently results in high quality dosimetry. Int J Radiat Oncol Biol Phys 2013; 85: 543-548.

10. Ghilezan M. Role of high dose rate brachytherapy in the treatment of prostate cancer. Cancer Radiother 2012; 16: 418-422.

11. Grills IS, Martinez AA, Hollander M et al. High dose rate brachytherapy as prostate cancer monotherapy reduces toxicity compared to low dose rate palladium seeds. J Urol 2004; 171: 1098-1104.

12. Galalae RM, Zakikhany NH, Geiger F et al. The 15-year outcomes of high-dose-rate brachytherapy for radical dose escalation in patients with prostate cancer. A benchmark for high-tech external beam radiotherapy alone? Brachytherapy 2014; 13: 117-122

13. Zamboglou N, Tselis N, Baltas D et al. High-dose-rate interstitial brachytherapy as monotherapy for clinically localized prostate cancer: treatment evolution and mature results. Int J Radiat Oncol Biol Phys 2013; 85: 672-678.

14. Hauswald H, Kamrava MR, Fallon JM et al. High-dose-rate monotherapy for localized prostate cancer: 10-year results. Int J Radiat Oncol Biol Phys 2015; 94: 667-674.

15. Krauss D, Kestin L, Ye H et al. Lack of benefit for the addition of androgen deprivation therapy to dose-escalated radiotherapy in the treatment of intermediate- and high-risk prostate cancer. Int J Radiat Oncol Biol Phys 2011; 80: 1064-1071.

16. Martinez AA, Pataki I, Edmundson G et al. Phase II prospective study of the use of conformal high-dose-rate brachytherapy as monotherapy for the treatment of favorable stage prostate cancer: A feasibility report. Int J Radiat Oncol Biol Phys 2001; 49: 61-69.

17. Yoshioka Y, Konishi K, Oh RJ et al. High-dose-rate brachytherapy without external beam irradiation for locally advanced prostate cancer. Radiother Oncol 2006; 80: 62-68.

18. Corner C, Rojas AM, Bryant L et al. A Phase II study of highdose-rate afterloading brachytherapy as monotherapy for the treatment of localized prostate cancer. Int J Radiat Oncol Biol Phys 2008; 72: 441-446.

19. Ghadjar P, Keller T, Rentsch CA et al. Toxicity and early treat- ment outcomes in low- and intermediate-risk prostate cancer managed by high-dose-rate brachytherapy as a monotherapy. Brachytherapy 2009; 8: 45-51. 
20. Rogers L, LeGrand R, Hopkins $S$ et al. Extended follow up of high-dose-rate brachytherapy as monotherapy for intermediate- risk prostate cancer. Brachytherapy 2010; 9: S55-S56.

21. D'Amico AV, Whittington R, Malkowicz SB et al. Biochemical outcome after radical prostatectomy, external beam radiation therapy, or interstitial radiation therapy for clinically localized prostate cancer. JAMA 1998; 280: 969-974.

22. Heidenreich A, Aus G, Bolla M et al. EAU guidelines on prostate cancer. Eur Urol 2008; 53: 68-80.

23. Mark R, Anderson P, Akins R et al. Interstitial high-dose-rate brachytherapy as monotherapy for early stage prostate cancer: Median 8-year results in 301 patients. Brachytherapy 2010; 9: 576.

24. Stock RG, Cesaretti JA, Stone NN. Disease-specific survival following the brachytherapy management of prostate cancer. Int J Radiat Oncol Biol Phys 2006; 64: 810-816.

25. Dearnaley DP, Sydes MR, Graham JD et al. Escalated-dose versus standard-dose conformal radiotherapy in prostate cancer: First results from the MRC RT01 randomised controlled trial. Lancet Oncol 2007; 8: 475-487.

26. Peeters ST, Heemsbergen WD, Koper PC et al. Dose-response in radiotherapy for localized prostate cancer: Results of the Dutch multicenter randomized phase III trial comparing 68 Gy of radiotherapy with 78 Gy. J Clin Oncol 2006; 24: 1990-1996.

27. Zietman AL, Bae K, Slater JD et al. Randomized trial comparing conventional-dose with high-dose conformal radiation therapy in early-stage adenocarcinoma of the prostate: long-term results from Proton Radiation Oncology Group/ American College of Radiology 95-09. J Clin Oncol 2010; 28: 1106-1111.

28. Viani GA, Stefano EJ, Afonso SL. Higher-than-conventional radiation doses in localized prostate cancer treatment: A meta-analysis of randomized, controlled trials. Int J Radiat Oncol Biol Phys 2009; 74: 1405-1418.

29. Michalski J, Moughan J, Purdy J. Initial results of a phase 3 randomized study of high dose 3DCRT/IMRT versus standard dose 3DCRT/IMRT in patients treated for localized prostate cancer. Int J Radiat Oncol Biol Phys 2014; 90: 1263.

30. Smith GD, Pickles T, Crook J et al. Brachytherapy improves biochemical failure-free survival in low- and intermediate-risk prostate cancer compared with conventionally fractionated external beam radiation therapy: A propensity score matched analysis. Int J Radiat Oncol Biol Phys 2015; 91: 505-516.

31. Grimm P, Billiet I, Bostwick D et al. Comparative analysis of prostate-specific antigen free survival outcomes for patients with low, intermediate and high risk prostate cancer treatment by radical therapy. Results from the Prostate Cancer Results Study Group: Cancer control rates: Comparison of treatment options. BJU Int 2012; 109: 22-29.

32. Chin J, Rumble RB, Kollmeier M et al. Brachytherapy for Patients With Prostate Cancer: American Society of Clinical Oncology/Cancer Care Ontario Joint Guideline Update. J Clin Oncol 2017; 35: 1737-1743.

33. Hauswald H, Kamrava MR, Fallon JM et al. High-dose-rate monotherapy for localized prostate cancer: 10-year results. Int J Radiat Oncol Biol Phys 2016; 94: 667-674.

34. Strouthos I, Tselis N, Chatzikonstantinou G et al. High dose rate brachytherapy as monotherapy for localised prostate cancer. Radiother Oncol 2018; 126: 270-277.

35. Zamboglou N, Tselis N, Baltas D et al. High-dose-rate interstitial brachytherapy as monotherapy for clinically localized prostate cancer: treatment evolution and mature results. Int I Radiat Oncol Biol Phys 2013; 85: 672-678.

36. Barkati M, Williams SG, Foroudi F et al. High-dose-rate brachytherapy as a monotherapy for favorable-risk prostate cancer: a Phase II trial. Int J Radiat Oncol Biol Phys 2012; 82 1889-1896.
37. Jawad MS, Dilworth JT, Gustafson GS et al. Outcomes associated with 3 treatment schedules of high-dose-rate brachytherapy monotherapy for favorable-risk prostate cancer. Int J Radiat Oncol Biol Phys 2016; 94: 657-666.

38. Krauss DJ, Ye H, Martinez AA et al. Favorable preliminary outcomes for men with low- and intermediate-risk prostate cancer treated with 19-Gy single-fraction high-dose-rate brachytherapy. Int J Radiat Oncol Biol Phys 2017; 97: 98-106.

39. Morton G, Chung HT, McGuffin M et al. Prostate high doserate brachytherapy as monotherapy for low and intermediate risk prostate cancer: Early toxicity and quality-of life results from a randomized phase II clinical trial of one fraction of 19 Gy or two fractions of 13.5 Gy. Radiother Oncol 2017; 122: 87-92.

40. Jones B, Dale RG. Mathematical models of tumour and normal tissue response. Acta Oncol 1999; 38: 883-893.

41. Tien CJ, Carlson DJ, Nath R, Chen ZJ. High-dose-rate brachytherapy as monotherapy for prostate cancer: the impact of cellular repair and source decay. Brachytherapy 2019; 18: 701-710.

42. Supiot S, Rousseau C, Dore M et al. Reoxygenation during radiotherapy in intermediate-risk prostate cancer. Radiother Oncol 2019; 133: 16-19.

43. Jones B, Tan LT, Dale RG. Derivation of the optimum dose per fraction from the linear quadratic model. Br J Radiol 1995; 68: 894-902.

44. Prada PJ, Cardenal J, Blanco AG et al. High-dose-rate interstitial brachytherapy as monotherapy in one fraction for the treatment of favorable stage prostate cancer: Toxicity and long-term biochemical results. Radiother Oncol 2016; 119: 411-416.

45. Morton G, McGuffin M, Chung HT et al. Prostate high doserate brachytherapy as monotherapy for low and intermediate risk prostate cancer: Efficacy results from a randomized phase II clinical trial of one fraction of $19 \mathrm{~Gy}$ or two fractions of 13.5 Gy. Radiother Oncol 2020; 146: 90-96. 